\title{
Hipertensão Arterial e trabalho entre docentes da educação básica da rede pública de ensino
}

\author{
Arterial Hypertension and work among teachers of basic education \\ in the public-school system
}

Marta Raquel Mendes Vieira (https://orcid.org/0000-0001-5185-5381) ${ }^{1}$ Tatiana Almeida de Magalhães (https://orcid.org/0000-0001-8371-863X) ${ }^{1}$ Rosângela Ramos Veloso Silva (https://orcid.org/0000-0003-3329-8133) ${ }^{1}$ Magda Mendes Vieira (https://orcid.org/0000-0001-5650-9787) ${ }^{1}$ Alfredo Maurício Batista de Paula (https://orcid.org/0000-0002-8715-0030) ${ }^{1}$ Vanessa Boaventura Araújo (https://orcid.org/0000-0001-7805-295X) ${ }^{1}$ Efigênia Ferreira e Ferreira (https://orcid.org/0000-0002-0665-211X) ${ }^{2}$ Desirée Sant'Ana Haikal (https://orcid.org/0000-0002-0331-0747) ${ }^{1}$
${ }^{1}$ Universidade Estadual de Montes Claros. Av. Prof. Rui Braga s/n, Vila Mauriceia. 39401-089 Montes Claros MG Brasil. martaraquelmendes@ hotmail.com

${ }^{2}$ Universidade Federal de Minas Gerais. Belo Horizonte MG Brasil

\begin{abstract}
The scope of this paper was to investigate the relationship between Arterial Hypertension $(A H)$ and the occupational profile of teachers of basic public education and present a theoretical model. A probabilistic sample was adopted by clusters, with $A H$ as the dependent variable. The independent variables were grouped in thematic blocks (Sociodemographic Characteristics, Occupational Profile, Behaviors and Health Outcomes) that composed the theoretical model. The analyses were corrected by the sample design. Hierarchical logistic regression was conducted. The prevalence of $A H$ was 25\%, and 58\% reported dissatisfaction with the work. There was a higher probability of AH among older teachers (OR $=3.7)$, without postgraduate qualification (OR $=1.4)$, who also worked in the private network $(O R=2.6)$, who had a higher salt intake $(O R=$ $1.7)$, with a high waist-hip ratio $(O R=1.9)$ and hypercholesterolemia / hypertriglyceridemia (OR $=1,5)$ and a lower chance among females $(O R=$ $0.5)$, who had other work activity $(O R=0.6)$ and were dissatisfied with the work $(O R=0.6)$. Thus, the occupational profile of teachers had an influence on $A H$. The relationship between work and teacher health demands attention and care, with measures that preserve and promote the health and well-being of teachers.
\end{abstract}

Key words Teachers, Workers' health, Arterial hypertension, Prevalence, Epidemiology
Resumo Objetivou-se investigar a relação entre Hipertensão Arterial (HA) e perfil ocupacional de docentes da educação básica pública e apresentar modelo teórico. Adotou-se amostra probabilística por conglomerados. A HA foi a variável dependente. As variáveis independentes foram agrupadas em blocos temáticos (Características Sociodemográficas, Perfil Ocupacional, Comportamentos e Desfechos em Saúde), que compuseram o modelo teórico. As análises foram corrigidas pelo desenho amostral. Foi conduzida regressão logística hierarquizada. A prevalência de HA foi de $25 \%$, enquanto que 58\% apresentaram insatisfação com o trabalho. Houve maior chance de HA entre os docentes mais velhos $(O R=3,7)$, sem pós-graduação $(O R=1,4)$, que atuavam também na rede privada $(O R=2,6)$, que apresentavam maior consumo de sal $(O R=1,7)$, com sobrepeso $(O R=2,1) /$ obesidade $(O R=7,2)$, com relação cintura-quadril elevada $(O R=1,9)$ e com hipercolesterolemia/hipertrigliceridemia $(O R=1,5)$ e menor chance entre os do sexo feminino $(O R=0,5)$, que exerciam outra atividade de trabalho $(O R=0,6)$ e insatisfeitos com o trabalho $(O R=0,6)$. Assim, o perfil ocupacional docente apresentou influência sobre a HA. A relação trabalho e saúde docente carece de atenção e cuidado, com adoção de medidas que preservem e promovam sua saúde e seu bem-estar. Palavras-chave Docentes, Saúde do trabalhador, Hipertensão arterial, Prevalência, Epidemiologia 


\section{Introdução}

O docente representa o elo na promoção do processo de ensino/aprendizagem ${ }^{1}$, complementa o papel da educação realizado pela família e é, muitas vezes, visto como modelo de comportamento a ser seguido por seus alunos². Porém, na docência, especificamente na educação básica, são muitos e significativos os desafios que acontecem em razão do alto grau de exigências sofridas e que podem acarretar em problemas de saúde física e mental ${ }^{3,4}$, podendo colocar o docente em risco diferenciado para determinadas doenças/condições, o que pode inclusive torná-lo incapacitado para o trabalho.

Segundo dados da Secretaria Estadual de Educação de São Paulo, em 2016 foram concedidas 128.178 licenças médicas a docentes dos anos finais do Ensino Fundamental e Ensino Médio, totalizando 2.901.529 dias de afastamento ${ }^{5}$. Em Minas Gerais, do total de afastamentos relacionados a servidores públicos da educação de 2001 a $2002,84,2 \%$ se referiu a docentes ${ }^{6}$. No Brasil, em estudo realizado entre 2015 e 2016 com docentes da educação básica, 53,3\% já faltaram ao trabalho ao menos um dia por motivo de saúde e 69,1\% independente do motivo. O absenteísmo por sete ou mais dias apresentou frequência de $14,7 \%{ }^{3}$. Os números refletem a sobrecarga a que são submetidos os doentes, o que certamente traz consequências para a aprendizagem dos alunos, podendo comprometer seu rendimento escolar ${ }^{2}$.

Problemas como o estresse ${ }^{4}$, Síndrome de burnout $^{7}$ e depressão ${ }^{8}$ têm sido apontados como prevalentes entre docentes da educação básica. Entretanto, poucos estudos vêm investigando a relação entre trabalho docente e Hipertensão Arterial (HA), apesar do impacto que essa condição patológica sistêmica crônica tem na saúde de muitas populações. A HA é apontada como importante causa de morte súbita, acidente vascular encefálico, infarto agudo do miocárdio, insuficiência cardíaca, doença arterial periférica e doença renal crônica, o que a torna um dos mais importantes fatores de risco cardiovascular. Em 2001, cerca de 7,6 milhões de mortes no mundo foram atribuídas à elevação da pressão arterial (PA), sendo a maioria em países de baixo e médio desenvolvimento econômico. No Brasil, doenças cardiovasculares representam a principal causa de morte na população adulta9, ${ }^{9}$.

Entretanto, não está suficientemente esclarecida na literatura a potencial relação existente entre o trabalho docente e a ocorrência de HA. Assim, este estudo teve como objetivo testar a relação entre HA e atuação docente na educação básica do ensino público. Para tanto, foi proposto um modelo teórico próprio que contemplasse, além dos fatores já reconhecidamente relacionados à HA, questões relativas especificamente ao trabalho docente.

\section{Método}

Estudo epidemiológico, transversal e analítico, conduzido com dados do Projeto ProfSMoc: "Condições crônicas de saúde e fatores associados entre professores da rede pública estadual de Montes Claros: estudo de base populacional". A cidade de Montes Claros representa o principal centro urbano da região norte do Estado de Minas Gerais, com características de capital regional e influência em todo o norte do estado e sul da Bahia. A população estimada para o município para 2017 foi de 402.027 habitantes $^{11}$.

\section{Amostragem}

A população do estudo foi composta por docentes de educação básica (ensinos fundamental e médio) da rede pública estadual. Foi conduzida amostragem probabilística por conglomerado em único estágio, no qual a escola foi considerada como Unidade Primária de Amostragem (UPA). Foram sorteadas 35 escolas (UPA), por probabilidade proporcional ao tamanho (PPT), dentre um total de 49 escolas estaduais da área urbana do município. O número de professores das escolas foi o parâmetro considerado como referência para o sorteio por PPT. Todos os docentes vinculados às escolas selecionadas que exerciam a docência há pelo menos um ano foram convidados a participar, sendo excluídos aqueles em desvio da função docente ou afastados do trabalho. A amostra estimada foi de 700 docentes, considerando população finita, prevalência de $50 \%$ do evento de interesse, nível de confiança de $95 \%$, erro tolerável de $5 \%$, acréscimo de $10 \%$ para compensação de perdas e deff $=2,0$.

\section{Estratégia de coleta de dados}

Foi realizado estudo piloto prévio, para teste e acerto do instrumento e da estratégia de coleta de dados. Os dados foram coletados de março a dezembro de 2016, com a realização de três etapas em cada escola. A primeira etapa foi o contato com a gestão da escola para apresentação do projeto, obtenção de consentimento 
da direção e agendamento de reunião com os docentes. Na segunda etapa ocorreu uma reunião com docentes para sensibilização e convite, realizada durante reunião de Módulo II (reuniões pedagógicas integrantes da carga horária do docente). Esse momento era destinado a entrega de questionários autoaplicáveis àqueles docentes que aceitavam participar da pesquisa, coleta do termo de consentimento e agendamento da terceira etapa. A terceira etapa foi destinada à devolução e conferência dos questionários preenchidos para evitar possíveis perdas de informações ou inconsistências e à realização de uma avaliação física. A avaliação física foi constituída por aferição dos seguintes parâmetros: peso, estatura, bioimpedância, circunferência da cintura (CC), circunferência do quadril (CQ) e PA. Esses procedimentos foram realizados de acordo com as recomendações da Organização Mundial da Saúde (OMS) com cumprimento de procedimentos padronizados ${ }^{12}$ e obedecendo as normas de biossegurança. Todos os avaliadores foram devidamente treinados e calibrados e atingiram concordância intra e interexaminadores satisfatórios (Kappa ponderado acima de $81 \%$ e Coeficiente de Correlação Intraclasse - CCI acima de 0,85$)$. As medidas foram todas aferidas duas vezes, adotando-se como resultado final a média das mensurações.

\section{Construção do modelo teórico}

Uma vez que não foi identificado modelo teórico que contemplasse a potencial influência de características do trabalho docente sobre a HA, foi proposto no presente estudo um modelo próprio, construído após extensa revisão da literatura que aborda o tema e predominantemente baseada em modelos prévios relativos a $\mathrm{HA}^{13,14}$ (Figura 1). Esse modelo retrata blocos de variáveis, hierarquicamente agrupados, numa relação direta ou indireta com o desfecho de interesse, a HA. O bloco de variáveis mais distal à HA foi o de Características Sociodemográficas, que contemplou características individuais que afetam a predisposição dos indivíduos. Posteriormente, no segundo nível, foi alocado o bloco de variáveis relativas ao Perfil Ocupacional Docente, considerado como possível fator de predisposição ao desfecho. Em seguida, num terceiro nível, o bloco de Comportamentos que contemplou Hábitos de Vida e Utilização dos Serviços de Saúde, sofrendo influência dos blocos que o antecederam e exercendo influência sobre os desfechos em saúde. Por fim, mais proximal à HA, o bloco espe- cífico de Desfechos em Saúde, com variáveis que retrataram Condições Subjetivas (sentidas pelos indivíduos) e Objetivas (aferidas por profissional). Na sequência, o desfecho final de interesse, a HA, recebendo influência hierárquica de todos os blocos anteriores (Figura 1).

\section{Variáveis do estudo}

Ao final, 42 variáveis independentes foram consideradas, sendo 36 variáveis provenientes do questionário e sete decorrentes da avaliação física.

A variável desfecho, a HA, foi construída considerando hipertensos aqueles docentes com medidas de PA sistólica superior a $140 \mathrm{mmHg}$, e/ ou PA diastólica superior a $90 \mathrm{mmHg}$ e/ou que relatassem uso de medicamento para controle da $\mathrm{PA}^{9}$. A PA foi aferida com o docente sentado em posição confortável, aguardando no mínimo 5 minutos para iniciar o procedimento. Todos os procedimentos adotados foram recomendados pelas Diretrizes Brasileiras de Hipertensão, em sua sexta versão ${ }^{10}$. A medida foi obtida no braço esquerdo com a palma da mão virada para cima. Foram tomadas duas medidas, com intervalo de 1 minuto entre elas (o valor final foi calculado pela média dos dois valores obtidos durate análise dos dados). $\mathrm{Na}$ aferição, utilizou-se esfigmomanômetro aneroide, previamente calibrado para garantir medições fieis e estetoscópio, ambos da marca $\mathrm{BIC}^{\circledR}$. O grau de concordância intra e inter examinadores foi obtido através do CCI, tendo sido obtido 0,915 para PA sistólica e de 0,935 para PA diastólica.

As demais variáveis consideradas, segundo os blocos de alocação, foram:

\section{Características sociodemográficas}

Variáveis Demográficas: Sexo, Idade, Estado civil e Cor da pele autodeclarada.

Variáveis Socioeconômicas: Classificação econômica coletada e analisada através do Critério Brasil, 2014 ${ }^{15}$ e Escolaridade (Com pós-graduação; Sem pós-graduação).

\section{Perfil Ocupacional Docente}

Foram considerados: Tempo de docência (20 anos ou menos; Acima de 20), Rede de atuação (Pública e privada; Somente pública), Jornada semanal de trabalho ( $\leq 20$ anos; 21-40anos; $>$ 40anos), Tipo de vínculo (Concursado/Efetivo; Contratado/Designado), Exerce outra atividade além da docência (Não; Sim) e Satisfação com o trabalho (Satisfeito; Insatisfeito). 


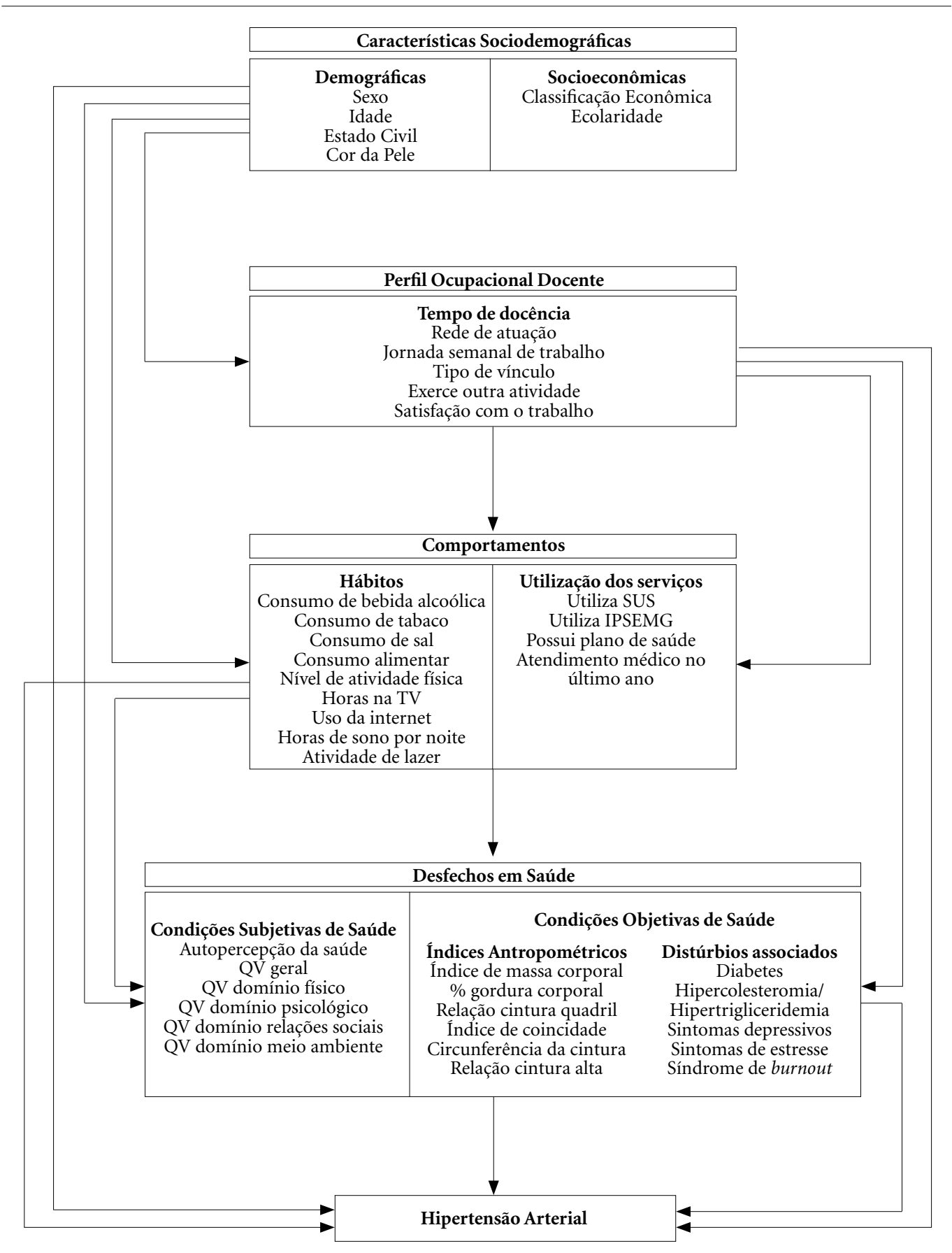

Figura 1. Modelo teórico Hipertensão Arterial Projeto ProfSMoc, 2016.

QV=qualidade de vida.

\section{Comportamentos}

Variáveis de Hábitos: Consumo de bebida alcoólica (Nunca/raramente; 1-2 vezes/semana; 3 ou mais vezes/semana) e Consumo de tabaco (Nunca fumou; Ex-fumante; Fumante), confor- me aferidas pela Vigilância de Fatores de Risco e Proteção para Doenças Crônicas por Inquérito Telefônico (VIGITEL) ${ }^{16}$, Consumo de sal autorrelatado, coletado segundo escala Likert e posteriormente dicotomizado (Adequado; Alto). 
Consumo alimentar (Adequado; Inadequado), que foi construído através da consolidação de sete questões por meio de análise de cluster. As questões utilizadas foram baseadas no VIGITEL ${ }^{16}$ : frequência (número de dias da semana) de ingestão de verduras/legumes; carnes com excesso de gorduras; fruta ou suco de fruta natural; refrigerante ou suco artificial; alimentos doces/ guloseimas; trocar a comida do almoço ou jantar por sanduíches, salgados, pizza ou outros lanches e, consumo de alimentos altamente processados. A análise revelou dois clusters capazes de discriminar padrões de comportamentos, sendo um com comportamentos mais adequados/satisfatórios e outro com comportamentos menos saudáveis (menor média semanal de consumo de verduras/legumes, fruta/suco natural; e maior média semanal de consumo de excesso de gorduras, refrigerante/suco artificial; alimentos doces/ guloseimas; substituição de refeições por lanches e o consumo de alimentos altamente processados). Nível de atividade física (Ativo; Sedentário/ Insuficientemente ativo), avaliado pelo Questionário Internacional de Atividade Física (IPAQ) na versão curta $^{17}$, Horas na TV (Não assiste TV; Assiste 2 hs ou menos; Assiste 3 hs ou mais), conforme VIGITEL ${ }^{16}$; Uso da internet (sem Adicção; Com Adicção), baseada no Internet Adiction Test (IAT), que possui 20 questões e permite um escore máximo de 100 pontos para classificação da dependência ao uso de internet ${ }^{18}$. Foi considerado com Adicção o docente que atingiu 40 pontos ou mais, sugerindo uso excessivo/ problemático da internet. Horas de sono por noite: variável coletada de forma numérica e posteriormente dicotomizada ( $\geq 7$ horas; $<6$ horas); Atividade de $\operatorname{lazer}^{16}$ (Sim; Não).

Variáveis de Utilização dos serviços: Utiliza o Sistema Único de Saúde - SUS (Sim; Não); Utiliza o Instituto de Previdência dos Servidores do Estado de Minas Gerais - IPSEMG (Sim; Não), que se trata de autarquia estadual que fornece assistência à saúde a seus segurados/dependentes e realiza a gestão de previdência social dos servidores do estado. As questões Possui plano de saúde (Sim; Não) e Atendimento médico no último ano (Sim; Não) foram coletadas conforme VIGITEL ${ }^{16}$ e posteriormente dicotomizadas.

\section{Desfechos em Saúde}

Variáveis de Condições subjetivas de saúde: Autopercepção da saúde (Excelente/Bom; Regular; Ruim/Péssimo) ${ }^{16}$, e Qualidade de vida - QV (Satisfatória; Insatisfatória). A QV foi aferida pelo instrumento WHOQOL-Bref da OMS, que possui 2 questões sobre QV geral e 24 que compõem quatro domínios (físico, psicológico, relações sociais e meio ambiente), sendo que, quanto maior o escore do domínio melhor a $\mathrm{QV}^{19}$. Os escores médios obtidos para QV geral e para cada domínio foram dicotomizados pelo limite inferior do seu Intervalo de 95\% de Confiança (IC-95\%), sendo que valores abaixo desse limite foram considerados com comprometimento na QV naquele domínio ou na QV geral.

Variáveis de Condições objetivas de saúde: divididas em Índices antropométricos e Distúrbios associados. Os Índices antropométricos mensurados foram: Índice de Massa Corporal - IMC (Normal; Sobrepeso; Obeso), calculado pela fórmula: $\mathrm{IMC}=$ peso $(\mathrm{Kg}) /$ estatura $^{2}(\mathrm{~m})$ e classificado com pontos de corte estabelecidos pela OMS, sendo: abaixo de $24,9 \mathrm{Kg} / \mathrm{m}^{2}$ normal, 25 a $29,9 \mathrm{Kg} / \mathrm{m}^{2}$ sobrepeso e $\geq 30 \mathrm{Kg} / \mathrm{m}^{2}$ obesidade $^{12}$. $\mathrm{O}$ peso dos docentes foi avaliado por balança digital calibrada (Modelo Digital Magna 150Kg, G Tech Ltda ${ }^{\circledR}$, São Paulo, SP) com aproximação de $0,1 \mathrm{~kg}$. A estatura foi mensurada com o auxílio de estadiômetro individual de base plana e aparato para nivelamento da altura funcionando como esquadro. Percentual de gordura corporal - \%GC (Normal; Elevado), obtido a partir da bioimpedância, método que estima o volume de água do corpo em conformidade com a resistência de corrente elétrica de baixa amplitude (500$800 \mathrm{~mA})$ e alta frequência $(50 \mathrm{kHz})$. Foi utilizado equipamento Bodystat ${ }^{\varpi}$, linha 1500 , aparelho com frequência tetrapolar, e eletrodos de gel para eletrocardiograma (Lectec Corporation ${ }^{\circledR}$, EUA). O protocolo de aferição seguiu o preconizado por Paiva et al. ${ }^{20}$. Foram considerados \%GC elevado valores acima de $30 \%$ para mulheres e acima de $20 \%$ para homens. Relação cintura quadril - RCQ (Normal x Elevado), obtida pela divisão da CC pela CQ (ambos em $\mathrm{cm}$ ). Os pontos de corte para se considerar a RCQ elevada são acima de 0,95 para homens e de 0,80 para mulheres. Índice de conicidade - IC (Normal x Elevado), obtido pela relação da Circunferência da Cintura (m) / [0, 109 X (Massa Corporal / Estatura)]). Os pontos de corte para IC elevado foram acima de 1.24 para homens e de 1.18 para mulheres. CC (Normal; Elevado) possui como classificação e pontos de corte: sem obesidade abdominal (< $88 \mathrm{~cm}$ para mulheres e $<102 \mathrm{~cm}$ para homens) e com obesidade abdominal ( $\geq 88 \mathrm{~cm}$ para mulheres e $\geq 102 \mathrm{~cm}$ para homens). Relação cintura altura - RCA (Normal; Elevado), obtida através da divisão do perímetro abdominal pela estatura (ambos em cm). Para homens ou mulheres o 
valor de 0,5 indica maior risco à saúde. Assim, se o perímetro abdominal é maior do que a metade do valor da estatura, maiores serão os riscos para condições crônicas de saúde.

Os Distúrbios associados investigados foram: Presença de diagnóstico prévio de Diabetes (Não; Sim) e Hipercolesterolemia/Hipertrigliceridemia (Não; Sim) conforme coletado pelo VIGITEL ${ }^{16,21}$. Sintomas depressivos (Ausência; Presença), avaliados pelo Inventário de Depressão de Beck $\mathrm{BDI}^{22}$, que contempla 21 questões e permite escore máximo de 63 pontos. Foram considerados com sintomas depressivos os docentes que fizeram 12 pontos ou mais ${ }^{23}$. Os Sintomas de estresse (Ausência; Presença) foram avaliados pelo Teste de Lipp ou ISSL - Inventário de Sintomas de Stress em Adulto, composto por 53 itens distribuídos em três dimensões: alerta; resistência; quase exaustão e exaustão. O diagnóstico positivo (sintomas de estresse) foi obtido quando a soma dos sintomas do inventário ultrapassou o ponto de corte limite para cada fase específica (Alerta/Alarme $>6$; Resistência/Luta > 3; Quase exaustão $>9$ e Exaustão $>8)^{24}$. A Síndrome de burnout - SB (Ausente; Presente) foi avaliada pelo CESQT (Cuestionário para la Evaluación del Síndrome de Quemarse por el Trabajo), composto por 20 questões e quatro dimensões: Ilusão pelo trabalho, Desgaste psíquico, Indolência e Culpa. Considerou-se na avaliação o escore global SB-Total, que considera 15 itens (a dimensão culpa nesse caso não é considerada). Nos docentes com SB-Total $<2$ a SB esteve ausente e naqueles com $\mathrm{SB}$-Total $\geq 2$, presente ${ }^{25}$.

\section{Análises conduzidas}

Todos os dados coletados foram digitados em duplicata, verificando-se a validade da digitação, com o objetivo de corrigir erros/falhas. Após a construção e a conferência do banco de dados, foram realizadas análises descritivas das variáveis, com apresentação de frequências absolutas e relativas (\%). As análises foram conduzidas respeitando-se a necessidade de correção pelo efeito de desenho em razão das variáveis serem provenientes de amostra por conglomerado. Para isso, foi atribuído um peso para cada indivíduo, correspondente ao inverso de sua probabilidade (p) de inclusão na amostra, considerando também a taxa de não resposta. Para o cálculo da probabilidade de inclusão dos professores foi adotada a expressão $\mathrm{p}=35 \mathrm{x}$ (número de professores da escola selecionada)/total de professores ${ }^{26}$.

Foram conduzidas análises bivariadas através do teste Qui-quadrado, sendo incluídas no mode- lo múltiplo apenas as variáveis que apresentaram valor-p $\leq 0,25$. Posteriormente, foi construído modelo múltiplo de forma hierarquizada, através de Regressão Logística Binária. A sequência de entrada e os níveis de organização dos blocos de variáveis seguiram o modelo teórico (Figura 1). Os blocos foram inseridos um a um e as variáveis de níveis mais distais com associação significativa com o desfecho foram mantidas para ajuste do modelo. Assim, o Modelo 1 considerou apenas as Características Sociodemográficas. O Modelo 2 considerou Características Sociodemográficas e as de Perfil ocupacional. O Modelo 3 considerou Características Sociodemográficas, de Perfil ocupacional e as de Comportamentos, enquanto o Modelo 4 considerou todos os blocos de variáveis.

A magnitude das associações foi estimada pela Razão das Chances (Odds Ratio - OR), considerando um nível de significância de 5\% ( $\leq 0,05)$. Para avaliar a qualidade de ajuste, utilizou-se o teste de Hosmer \& Lemeshow e o pseudo $\mathrm{R}^{2}$. Os dados foram analisados com auxílio do programa Statistical Package for Social Sciences (SPSS), versão 18.0. Para correção pelo desenho amostral, foi utilizado o módulo Complex Sample.

\section{Questões éticas}

O Projeto ProfSMoc foi aprovado e recomendado pelas Secretarias Regional e Estadual de Educação. Foram atendidos todos os princípios éticos do Conselho Nacional de Saúde (CNS) / Resolução n466/2012 e da Declaração de Helsinque da Associação Médica Mundial. O projeto foi aprovado pelo Comitê de Ética em Pesquisa (CEP) da Unimontes. Todos os gestores e docentes das escolas participantes assinaram o Termo de Consentimento Livre e Esclarecido. Aqueles com suspeita de problemas físicos e/ou psicológicos foram encaminhados a serviços de referência públicos parceiros do projeto.

\section{Resultados}

O Projeto ProfSMoc obteve dados de 760 profissionais da rede pública de ensino, porém, o presente trabalho foi conduzido entre 710 docentes conforme critérios de exclusão (15 não atuantes em sala de aula e 35 sem informação sobre HA). Após a ponderação atribuída a cada docente, a fim de corrigir as estimativas pelo desenho amostral, foi encontrado peso mínimo de 0,76 e máximo de 3,53 (amplitude 2,77).

Observou-se prevalência de 24,9\% de hipertensos. A média de idade dos docentes foi de 40,5 
$( \pm 9,6)$ anos. Houve predomínio do sexo feminino $(86 \%)$, da Classe social B $(62 \%)$, com menos de 20 anos de atuação docente (81\%), insatisfeitos com o trabalho (58\%), com relato de consumo de sal adequado (77\%), que passaram por atendimento médico no último ano (87\%) e com autopercepção positiva da saúde (67\%). Cerca de $53 \%$ apresentaram sobrepeso ou obesidade, $25 \%$ relataram hipercolesterolemia e/ou hipertrigliceridemia, $40 \%$ com sintomas de estresse e 13\% com Síndrome de burnout (Tabela 1).
$\mathrm{Na}$ análise bivariada, 26 variáveis apresentaram associação com o desfecho ao nível de $25 \%$ de significância e foram selecionadas para iniciar a modelagem múltipla (Tabela 1 ).

A análise múltipla hierarquizada revelou que a chance de HA entre docentes sofreu influencia do sexo, da idade, da escolaridade, do fato de exercer ou não outra atividade de trabalho, da satisfação com o trabalho, da rede de ensino em que atua, do consumo de sal, do IMC, da RCQ e da hipercolesterolemia/hipertrigliceridemia (Ta-

Tabela 1. Distribuição dos docentes de Ensino Fundamental e Médio da Rede Pública Estadual (n= 710) e análise bivariada dos fatores relacionados à Hipertensão Arterial conforme grupo de variáveis do Modelo Teórico proposto. Montes Claros (MG), 2016.

\begin{tabular}{|c|c|c|c|c|c|c|c|}
\hline \multirow{3}{*}{ Variável } & \multirow{2}{*}{\multicolumn{2}{|c|}{ Total }} & \multicolumn{4}{|c|}{ Hipertensão Arterial } & \multirow{3}{*}{ p valo } \\
\hline & & & \multicolumn{2}{|c|}{ Não Hipertenso } & \multicolumn{2}{|c|}{ Hipertenso } & \\
\hline & $\mathbf{n}$ & $\%^{ \pm}$ & $\mathbf{n}$ & $\%$ & $\mathrm{n}$ & $\%$ & \\
\hline \multicolumn{8}{|c|}{ Características Sociodemográficas } \\
\hline \multicolumn{8}{|l|}{ Demográficas } \\
\hline Sexo & & & & & & & 0,050 \\
\hline Masculino & 118 & 14,3 & 80 & 67,8 & 38 & 32,2 & \\
\hline Feminino & 592 & 85,7 & 452 & 76,4 & 140 & 23,6 & \\
\hline Idade (em anos) & & & & & & & 0,000 \\
\hline$\leq 40$ & 358 & 50,1 & 308 & 86,0 & 50 & 14,0 & \\
\hline$>41$ & 352 & 49,9 & 224 & 63,6 & 128 & 36,4 & \\
\hline Estado civil & & & & & & & 0,045 \\
\hline Casado/União estável & 439 & 61,3 & 333 & 75,9 & 106 & 24,1 & \\
\hline Solteiro & 191 & 27,7 & 148 & 77,5 & 43 & 22,5 & \\
\hline Divorciado/Viúvo & 80 & 11,0 & 51 & 63,8 & 29 & 36,3 & \\
\hline Cor da pele ${ }^{\star}$ & & & & & & & 0,226 \\
\hline Branca/Amarela & 230 & 31,6 & 168 & 73,0 & 62 & 27,0 & \\
\hline Negra & 66 & 9,5 & 45 & 68,2 & 21 & 31,8 & \\
\hline Parda/Indígena & 413 & 58,9 & 318 & 77,0 & 95 & 23,0 & \\
\hline \multicolumn{8}{|l|}{ Socioeconômicas } \\
\hline Classificação econômica & & & & & & & 0,574 \\
\hline Classe A & 80 & 11,1 & 57 & 71,3 & 23 & 28,8 & \\
\hline Classe B & 430 & 62,2 & 322 & 74,9 & 108 & 25,1 & \\
\hline Classes C e D/E & 189 & 26,6 & 146 & 77,2 & 43 & 22,8 & \\
\hline Escolaridade & & & & & & & 0,016 \\
\hline Com pós-graduação & 428 & 55,1 & 309 & 78,4 & 85 & 21,6 & \\
\hline Sem pós-graduação & 332 & 44,9 & 223 & 70,6 & 93 & 29,4 & \\
\hline \multicolumn{8}{|l|}{ Perfil Ocupacional Docente } \\
\hline Tempo de docência (anos) & & & & & & & 0,003 \\
\hline$\leq 20$ ou menos & 569 & 80,8 & 440 & 77,3 & 129 & 22,7 & \\
\hline Acima de 20 & 140 & 19,2 & 91 & 65,0 & 49 & 35,0 & \\
\hline Rede de atuação & & & & & & & 0,005 \\
\hline Pública e privada & 65 & 8,5 & 58 & 89,2 & 7 & 10,8 & \\
\hline Somente pública & 645 & 91,5 & 474 & 73,5 & 171 & 26,5 & \\
\hline
\end{tabular}


Tabela 1. Distribuição dos docentes de Ensino Fundamental e Médio da Rede Pública Estadual (n = 710) e análise bivariada dos fatores relacionados à Hipertensão Arterial conforme grupo de variáveis do Modelo Teórico proposto. Montes Claros (MG), 2016.

\begin{tabular}{|c|c|c|c|c|c|c|c|}
\hline \multirow{3}{*}{ Variável } & \multirow{2}{*}{\multicolumn{2}{|c|}{ Total }} & \multicolumn{4}{|c|}{ Hipertensão Arterial } & \multirow{3}{*}{ p valor } \\
\hline & & & \multicolumn{2}{|c|}{ Não Hipertenso } & \multicolumn{2}{|c|}{ Hipertenso } & \\
\hline & $\mathbf{n}$ & $\%^{¥}$ & $\mathbf{n}$ & $\%$ & $\mathbf{n}$ & $\%$ & \\
\hline Jornada semanal de trabalho (em hs) & & & & & & & 0,341 \\
\hline$\leq 20$ & 269 & 38,4 & 207 & 77,0 & 62 & 23,0 & \\
\hline $21-40$ & 370 & 51,9 & 269 & 72,7 & 101 & 27,3 & \\
\hline$>40$ & 71 & 9,7 & 56 & 78,9 & 15 & 21,1 & \\
\hline Tipo de vínculo* & & & & & & & 0,942 \\
\hline Concursado/Efetivo & 313 & 40,8 & 234 & 74,8 & 79 & 25,2 & \\
\hline Contratado/Designado & 396 & 59,2 & 297 & 75,0 & 99 & 25,0 & \\
\hline Exerce outra atividade & & & & & & & 0,039 \\
\hline Não & 555 & 78,4 & 406 & 73,2 & 149 & 26,8 & \\
\hline Sim & 155 & 21,6 & 126 & 81,3 & 29 & 18,7 & \\
\hline Satisfação com o trabalho & & & & & & & 0,021 \\
\hline Satisfeito & 287 & 42,2 & 202 & 70,4 & 85 & 29,6 & \\
\hline Insatisfeito & 423 & 57,8 & 330 & 78,0 & 93 & 22,0 & \\
\hline \multicolumn{8}{|l|}{ Comportamentos } \\
\hline \multicolumn{8}{|l|}{ Hábitos } \\
\hline Consumo de bebida alcoólica & & & & & & & 0,363 \\
\hline Nunca/Raramente & 575 & 80,9 & 437 & 76,0 & 138 & 24,0 & \\
\hline $1-2$ vezes/semana & 114 & 16,6 & 81 & 71,1 & 33 & 28,9 & \\
\hline 3 ou mais vezes/ semana & 21 & 2,5 & 14 & 66,7 & 7 & 33,3 & \\
\hline Consumo de tabaco & & & & & & & 0,001 \\
\hline Nunca fumou & 625 & 88,1 & 481 & 77,0 & 144 & 23,0 & \\
\hline Ex-fumante & 73 & 10,1 & 42 & 57,5 & 31 & 42,5 & \\
\hline Fumante & 12 & 1,8 & 9 & 75,0 & 3 & 25,0 & \\
\hline Consumo de sal & & & & & & & 0,084 \\
\hline Adequado & 548 & 77,1 & 419 & 76,5 & 129 & 23,5 & \\
\hline Alto & 162 & 22,9 & 113 & 69,8 & 49 & 30,2 & \\
\hline Consumo alimentar & & & & & & & 0,628 \\
\hline Adequado & 456 & 64,3 & 339 & 74,3 & 117 & 25,7 & \\
\hline Inadequado & 254 & 35,7 & 193 & 76,0 & 61 & 24,0 & \\
\hline Nível de atividade física* & & & & & & & 0,111 \\
\hline Ativo & 342 & 49,4 & 247 & 72,2 & 95 & 27,8 & \\
\hline $\begin{array}{l}\text { Sedentário/Insuficientemente } \\
\text { ativo }\end{array}$ & 359 & 50,6 & 278 & 77,4 & 81 & 22,6 & \\
\hline Horas na $\mathrm{TV}^{\star}$ & & & & & & & 0,188 \\
\hline Não assiste TV & 72 & 10,0 & 57 & 79,2 & 15 & 20,8 & \\
\hline 2 hs ou menos & 495 & 70,3 & 376 & 76,0 & 119 & 24,0 & \\
\hline 3 hs ou mais & 140 & 19,6 & 97 & 69,3 & 43 & 30,7 & \\
\hline Uso da internet ${ }^{*}$ & & & & & & & 0,763 \\
\hline Sem Adicção & 650 & 92,7 & 488 & 75,1 & 162 & 24,9 & \\
\hline Com Adicção & 51 & 7,3 & 40 & 78,0 & 11 & 22,0 & \\
\hline Horas de sono por noite & & & & & & & 0,248 \\
\hline$\geq 7$ & 328 & 46,7 & 239 & 72,9 & 89 & 27,1 & \\
\hline$<6$ & 381 & 53,3 & 292 & 76,6 & 89 & 23,4 & \\
\hline Atividade de lazer* & & & & & & & 0,900 \\
\hline Sim & 433 & 61,7 & 325 & 75,1 & 108 & 24,9 & \\
\hline Não & 276 & 38,3 & 206 & 74,6 & 70 & 25,4 & \\
\hline
\end{tabular}


Tabela 1. Distribuição dos docentes de Ensino Fundamental e Médio da Rede Pública Estadual (n=710) e análise bivariada dos fatores relacionados à Hipertensão Arterial conforme grupo de variáveis do Modelo Teórico proposto. Montes Claros (MG), 2016.

\begin{tabular}{|c|c|c|c|c|c|c|c|}
\hline \multirow{3}{*}{ Variável } & \multirow{2}{*}{\multicolumn{2}{|c|}{ Total }} & \multicolumn{4}{|c|}{ Hipertensão Arterial } & \multirow{3}{*}{ p valor } \\
\hline & & & \multicolumn{2}{|c|}{ Não Hipertenso } & \multicolumn{2}{|c|}{ Hipertenso } & \\
\hline & $\mathbf{n}$ & $\%^{¥}$ & $\mathbf{n}$ & $\%$ & $\mathbf{n}$ & $\%$ & \\
\hline \multicolumn{8}{|l|}{ Utilização dos Serviços } \\
\hline Utiliza SUS ${ }^{\star}$ & & & & & & & 0,299 \\
\hline Sim & 281 & 40,1 & 205 & 73,0 & 76 & 27,0 & \\
\hline Não & 428 & 59,9 & 327 & 76,4 & 101 & 23,6 & \\
\hline Utiliza IPSEMG ${ }^{\star}$ & & & & & & & 0,280 \\
\hline Sim & 482 & 67,9 & 355 & 73,7 & 127 & 26,3 & \\
\hline Não & 226 & 32,1 & 175 & 77,4 & 51 & 22,6 & \\
\hline Possui plano de saúde & & & & & & & 0,403 \\
\hline $\operatorname{Sim}$ & 202 & 29,0 & 147 & 72,8 & 55 & 27,2 & \\
\hline Não & 508 & 71,0 & 385 & 75,8 & 123 & 24,2 & \\
\hline Atendimento médico no último ano* & & & & & & & 0,064 \\
\hline Sim & 612 & 87,1 & 451 & 73,7 & 161 & 26,3 & \\
\hline Não & 97 & 12,9 & 80 & 82,5 & 17 & 17,5 & \\
\hline \multicolumn{8}{|l|}{ Desfechos em Saúde } \\
\hline \multicolumn{8}{|l|}{ Condições Subjetivas de Saúde } \\
\hline Autopercepção da saúde & & & & & & & 0,000 \\
\hline Excelente/Bom & 467 & 66,9 & 369 & 79,0 & 98 & 21,0 & \\
\hline Regular & 205 & 27,9 & 142 & 69,3 & 63 & 30,7 & \\
\hline Ruim/Péssimo & 38 & 5,2 & 21 & 55,3 & 17 & 44,7 & \\
\hline QV geral & & & & & & & 0,069 \\
\hline Satisfatória & 369 & 52,2 & 287 & 77,8 & 82 & 22,2 & \\
\hline Insatisfatória & 341 & 47,8 & 245 & 71,8 & 96 & 28,2 & \\
\hline QV domínio físico & & & & & & & 0,411 \\
\hline Satisfatória & 388 & 55,5 & 286 & 73,7 & 102 & 26,3 & \\
\hline Insatisfatória & 322 & 44,5 & 246 & 76,4 & 76 & 23,6 & \\
\hline QV domínio psicológico & & & & & & & 0,994 \\
\hline Satisfatória & 415 & 58,7 & 311 & 74,9 & 104 & 25,1 & \\
\hline Insatisfatória & 295 & 41,3 & 221 & 74,9 & 74 & 25,1 & \\
\hline QV domínio relações sociais & & & & & & & 0,575 \\
\hline Satisfatória & 386 & 55,0 & 286 & 74,1 & 100 & 25,9 & \\
\hline Insatisfatória & 324 & 45,0 & 246 & 75,9 & 78 & 24,1 & \\
\hline QV domínio meio ambiente & & & & & & & 0,634 \\
\hline Satisfatória & 356 & 50,8 & 264 & 74,2 & 92 & 25,8 & \\
\hline Insatisfatória & 354 & 49,2 & 268 & 75,7 & 86 & 24,3 & \\
\hline \multicolumn{8}{|l|}{ Condições Objetivas de Saúde } \\
\hline \multicolumn{8}{|l|}{ Índices Antropométricos } \\
\hline Índice de massa corporal & & & & & & & 0,000 \\
\hline Normal & 329 & 46,9 & 293 & 89,1 & 36 & 10,9 & \\
\hline Sobrepeso & 250 & 36,6 & 180 & 72,0 & 70 & 28,0 & \\
\hline Obeso & 113 & 16,5 & 51 & 45,1 & 62 & 54,9 & \\
\hline$\%$ gordura corporal $^{\star}$ & & & & & & & 0,008 \\
\hline Normal & 70 & 10,6 & 62 & 88,6 & 8 & 11,4 & \\
\hline Elevado & 627 & 89,4 & 465 & 74,2 & 162 & 25,8 & \\
\hline Relação cintura quadril ${ }^{*}$ & & & & & & & 0,000 \\
\hline Normal & 575 & 85,0 & 466 & 81,0 & 109 & 19,0 & \\
\hline Elevado & 104 & 15,0 & 48 & 46,2 & 56 & 53,8 & \\
\hline
\end{tabular}


Tabela 1. Distribuição dos docentes de Ensino Fundamental e Médio da Rede Pública Estadual (n=710) e análise bivariada dos fatores relacionados à Hipertensão Arterial conforme grupo de variáveis do Modelo Teórico proposto. Montes Claros (MG), 2016.

\begin{tabular}{|c|c|c|c|c|c|c|c|}
\hline \multirow{3}{*}{ Variável } & \multirow{2}{*}{\multicolumn{2}{|c|}{ Total }} & \multicolumn{4}{|c|}{ Hipertensão Arterial } & \multirow{3}{*}{ p valor } \\
\hline & & & \multicolumn{2}{|c|}{ Não Hipertenso } & \multicolumn{2}{|c|}{ Hipertenso } & \\
\hline & $\mathbf{n}$ & $\%^{\mp}$ & $\mathbf{n}$ & $\%$ & $\mathbf{n}$ & $\%$ & \\
\hline Índice de conicidade & & & & & & & 0,000 \\
\hline Normal & 567 & 83,5 & 457 & 80,6 & 110 & 19,4 & \\
\hline Elevado & 112 & 16,5 & 56 & 50,0 & 56 & 50,0 & \\
\hline Circunferência da cintura ${ }^{*}$ & & & & & & & 0,000 \\
\hline Normal & 599 & 85,4 & 485 & 81,0 & 114 & 19,0 & \\
\hline Elevado & 102 & 14,6 & 45 & 44,1 & 57 & 55,9 & \\
\hline Relação cintura altura* & & & & & & & 0,000 \\
\hline Normal & 408 & 62,4 & 353 & 86,5 & 55 & 13,5 & \\
\hline Elevado & 247 & 37,6 & 139 & 56,3 & 108 & 43,7 & \\
\hline \multicolumn{8}{|l|}{ Distúrbios Associados } \\
\hline Diabetes $^{*}$ & & & & & & & 0,083 \\
\hline Não & 690 & 97,3 & 520 & 75,4 & 170 & 24,6 & \\
\hline Sim & 19 & 2,7 & 11 & 57,9 & 8 & 42,1 & \\
\hline $\begin{array}{l}\text { Hipercolesterolemia/ } \\
\text { Hipertrigliceridemia }\end{array}$ & & & & & & & 0,000 \\
\hline Não & 535 & 74,8 & 428 & 80,0 & 107 & 20,0 & \\
\hline Sim & 175 & 25,2 & 104 & 59,4 & 71 & 40,6 & \\
\hline Sintomas depressivos ${ }^{\star}$ & & & & & & & 0,641 \\
\hline Ausência & 547 & 76,9 & 408 & 74,6 & 139 & 25,4 & \\
\hline Presença & 161 & 23,1 & 123 & 76,4 & 38 & 23,6 & \\
\hline Sintomas de estresse ${ }^{\star}$ & & & & & & & 0,566 \\
\hline Ausência & 422 & 60,0 & 319 & 75,6 & 103 & 24,4 & \\
\hline Presença & 285 & 40,0 & 210 & 73,7 & 75 & 26,3 & \\
\hline Síndrome de burnout & & & & & & & 0,039 \\
\hline Ausente & 609 & 86,6 & 448 & 73,6 & 161 & 26,4 & \\
\hline Presente & 101 & 13,4 & 84 & 83,2 & 17 & 16,8 & \\
\hline
\end{tabular}

bela 2). O pseudo $\mathrm{R}^{2}$ obtido no modelo final foi de $33,2 \%$ e o Teste de Hosmer e Lemeshow mostrou-se não significativo ( $\mathrm{p}$ valor $=0,720$ ).

O modelo final ajustado apresentou variáveis independentes provenientes de todos os blocos propostos no modelo teórico, sendo que o bloco relativo ao Perfil Ocupacional Docente contribuiu, no modelo final ajustado, com três variáveis (rede de ensino em que o decente atua, se exerce atividade de trabalho além da docência e satisfação com o trabalho docente).

\section{Discussão}

Cerca de um quarto dos docentes da educação básica avaliados apresentou HA. Os docentes com maior chance de HA foram os mais velhos, sem pós-graduação, com atuação conjunta na rede pública e privada, com alto consumo de sal, com sobrepeso ou obesidade, com a RCQ elevada e com hipercolesterolemia e/ou hipertrigliceridemia. Os docentes com menor chance de HA foram os do sexo feminino, que exerciam outra atividade de trabalho além da docência e os insatisfeitos com o trabalho. Três variáveis relativas ao Perfil Ocupacional Docente revelaram-se associadas a HA, mesmo após ajuste pelas demais variáveis.

O modelo teórico proposto apresentou pertinência uma vez que no modelo final ficaram retidas variáveis provenientes de todos os quatro blocos considerados. Este modelo deu conta de explicar 33,2\% da variabilidade de ocorrência de HA entre docentes. Além disso, a análise hierarquizada permitiu identificar a contribuição 
Tabela 2. Modelo final da Análise de Regressão Logística Binária Hierarquizada dos fatores associados à Hipertensão Arterial em docentes do Ensino Fundamental e Médio da Rede Pública Estadual. Montes Claros (MG), 2016.

\begin{tabular}{|c|c|c|c|}
\hline Modelos & Variáveis & OR (IC95\%) & p valor \\
\hline Modelo 1 & Sexo & & \\
\hline \multirow[t]{8}{*}{ Características Sociodemográficas } & Masculino & 1 & \\
\hline & Feminino & $0,539(0,341-0,854)$ & 0,008 \\
\hline & Idade (em anos) & & \\
\hline & $\leq 40$ & 1 & \\
\hline & $>41$ & $3,757(2,573-5,488)$ & 0,000 \\
\hline & Escolaridade & & \\
\hline & Com Pós-graduação & 1 & \\
\hline & Sem Pós-graduação & $1,435(1,005-2,048)$ & 0,047 \\
\hline \multicolumn{4}{|l|}{ Pseudo R²Modelo $1=0,120$} \\
\hline \multirow{9}{*}{$\begin{array}{l}\text { Modelo } 2 \\
\text { Características Sociodemográficas } \\
\text { Perfil ocupacional docente }\end{array}$} & Exerce outra atividade de trabalho & & \\
\hline & Não & 1 & \\
\hline & Sim & $0,615(0,383-0,988)$ & 0,045 \\
\hline & Satisfação com o trabalho & & \\
\hline & Satisfeito & 1 & \\
\hline & Insatisfeito & $0,654(0,455-0,940)$ & 0,022 \\
\hline & Rede de ensino em que atua & & \\
\hline & Somente pública & 1 & \\
\hline & Pública e privada & $2,683(1,159-6,209)$ & 0,021 \\
\hline \multicolumn{4}{|l|}{ Pseudo $\mathrm{R}^{2}$ Modelo $2=0,152$} \\
\hline Modelo 3 & Consumo de sal & & \\
\hline Características Sociodemográficas & Adequado & 1 & \\
\hline $\begin{array}{l}\text { Perfil ocupacional docente } \\
\text { Comportamentos }\end{array}$ & Alto & $1,748(1,142-2,677)$ & 0,010 \\
\hline \multicolumn{4}{|l|}{ Pseudo $\mathrm{R}^{2}$ Modelo $3=0,164$} \\
\hline \multirow{10}{*}{$\begin{array}{l}\text { Modelo } 4 \\
\text { Características Sociodemográficas } \\
\text { Perfil ocupacional docente } \\
\text { Comportamentos } \\
\text { Desfechos em saúde }\end{array}$} & IMC & & \\
\hline & Normal & 1 & \\
\hline & Sobrepeso & $2,118(1,300-3,449)$ & 0,003 \\
\hline & Obeso & $\begin{array}{r}7,232(4,021- \\
13,008)\end{array}$ & 0,000 \\
\hline & Relação Cintura Quadril & & \\
\hline & Normal & 1 & \\
\hline & Elevado & $1,936(1,136-3,300)$ & 0,015 \\
\hline & Hipercolesterolemia/Hipertrigliceridemia & & \\
\hline & Não & 1 & \\
\hline & Sim & $1,503(0,944-2,393)$ & 0,086 \\
\hline
\end{tabular}

de cada bloco na explicação da variabilidade do desfecho, sendo que as Condições Objetivas de Saúde, incluídas no bloco Desfechos sem Saúde, foram as que mais contribuíram com tal explicação.

A prevalência de HA verificada entre os docentes deste estudo (24,9\%) foi compatível com o observado na população adulta brasileira em $2016(25,7 \%)^{27}$. Estudos prévios realizados exclusivamente com docentes mostraram prevalências um pouco menores, variando de $16 \%$ em $2011^{28}$ a $20 \%$ em $2013 / 2015^{29,30}$. Esse aparente incremento na prevalência de HA ao longo do tempo é consistente com dados do VIGITEL, no qual se observou aumento da frequência média de diagnóstico de HA em 0,35 pontos percentuais ao ano entre os brasileiros, considerando o período de 2012 a $2016^{27}$.

Na presente investigação, os docentes do sexo masculino apresentaram maior chance de HA, 
conforme também observado entre os universitários ${ }^{28,31}$; e diferindo de estudos conduzidos com a população em geral $^{32,33}$. A maior chance de HA entre os mais velhos já está bastante documentada na literatura ${ }^{27,28,30,32,33}$. A maior chance de HA entre os docentes sem pós-graduação corrobora com estudos prévios que identificaram menor escolaridade associada à maior ocorrência de HA na população em geral $^{27,33,34}$.

Atuar concomitantemente na rede pública e privada de ensino aumentou as chances de HA. Por outro lado, possuir outro vínculo de trabalho diferente da docência, diminuiu as chances de HA. A baixa remuneração da categoria ${ }^{2}$ poderia contribuir para o acúmulo de cargos de trabalhado. Nesse sentido, foi interessante constatar que exercer um segundo cargo na docência aumentou a chance de HA, enquanto que exercer um segundo cargo fora da docência operou como proteção à HA. Reconhece-se que a prática docente é realizada em meio a tensões, com grande responsabilidade dos docentes, e percebida como um trabalho complexo, que envolve problemáticas diversas, seja do sistema educacional ou aquelas vivenciadas diariamente em sala de aula e que impactam na saúde do docente ${ }^{35}$. Assim, sugere-se que ao se deparar com um universo de trabalho diferente, o docente poderia revigorar sua energia, diminuindo a chance de ocorrência de HA, diferentemente daqueles docentes que possuem um segundo cargo de atuação também docente.

Outro achado que merece reflexão, ainda no bloco relativo ao Perfil Ocupacional Docente, refere-se à menor chance de HA entre os docentes insatisfeitos com o trabalho. Já foi observada correlação positiva significativa entre satisfação no trabalho e capacidade para o trabalho entre docentes universitários ${ }^{36}$. A maior dedicação ao trabalho inevitavelmente acarretaria em maior frequência de exposição a fatores adversos potencialmente responsáveis por tensão, como maior desapontamento diante de comportamentos e/ou rendimento indesejável dos alunos, maior frustração diante de situações fora de sua governabilidade, maior acúmulo de responsabilidades e/ou funções, maior autocobrança no cumprimento de horários e prazos, necessidade de dedicação em finais de semana ou no tempo livre ${ }^{37}$, que podem não ser suficientes para tornar o docente desiludido ou insatisfeito com a profissão, mas podem adoecê-lo a longo prazo. Estudo prévio verificou que entre docentes insatisfeitos, a exoneração do cargo ou pelo menos a redução da sua jornada de trabalho é algo almejado ${ }^{38}$, demostrando o enfraquecimento do vínculo do- cente-trabalho, com maior apatia e/ou negligencia diante das questões relativas ao seu cargo. A postura "descomprometida" protegeria o docente do estresse, que comumente é referenciado como próprio da profissão ${ }^{37}$, e evitaria o desenvolvimento da HA.

A relação entre HA e o maior consumo de sal já está estabelecida na literatura ${ }^{34,39}$. No Brasil, o consumo de sal pela população costuma ser acima do recomendado, apesar de estar havendo uma tendência de redução de seu teor nos alimentos industrializados ${ }^{40}$.

Foi verificado na literatura associação estatisticamente significativa da HA com IMC ${ }^{28,30,41}$, dislipidemia $^{30}$, hipercolesterolemia $(\mathrm{OR}=1,9)^{34}$, diabetes mellitus ${ }^{30}$, circunferência abdominal $(\mathrm{RP}=2,53)$, percentual de gordura corporal ( $\mathrm{RP}$ $=3,01)$, triacilglicerois $(\mathrm{RP}=3,93)^{28}$ e excesso de peso $(\mathrm{RP}=1,47)^{42}$. No presente estudo, especificamente sobre as Condições Objetivas de Saúde, foi observada associação da HA com IMC, RCQ e hipercolesterolemia/ hipertrigliceridemia, variáveis do bloco que mais contribuiu na explicação do desfecho. O IMC elevado está associado a ganho de peso, obesidade abdominal e desenvolvimento de doenças cardiovasculares ${ }^{43}$, dentre elas a HA. Práticas como orientações nutricionais, orientações em saúde e realização de exercícios físicos podem atuar na redução de níveis pressóricos, níveis de lipoproteína de alta densidade, na $\mathrm{CQ}$ e na $\mathrm{RCQ}^{44}$. Com isso, deve-se fomentá-las entre docentes, tanto no campo teórico quanto prático, enfatizando a importância de tais ações voltadas a esses profissionais.

O presente estudo apresenta como restrição o fato de ser transversal, limitando-se a inferência causal. O percentual da variância da HA explicado no modelo final foi modesto $(33,2 \%)$, sugerindo que há ainda outros fatores não investigados que têm relação com a HA e que precisam de maior elucidação, com inclusão de outras variáveis individuais e de variáveis contextuais, relativas aos modos de vida e condições de trabalho. Além disso, o autorrelato também foi fonte de dados, coletados através de questionário autoaplicado.

Mas embora o estudo tenha apresentado limitações, também foram observadas questões positivas, que conferiram qualidade ao trabalho realizado como o grande número de variáveis independentes investigadas, a realização da avaliação física com aferição de parâmetros de saúde, a realização de treinamento/ calibração dos examinadores, a digitação dos dados em duplicata, que conferiram maior validade aos dados analisados. Além disso, o modelo teórico construído 
pode ser considerado pertinente, abrangente e inovador por considerar questões da atividade de trabalho docente e a sua relação com HA, permitindo confirmar que esses dois campos apresentam associação. Em acréscimo a isso, a análise hierarquizada possibilitou conhecer o incremento de contribuição de cada bloco temático sobre o desfecho.

Ressalta-se que o trabalho pode e deve ser um ambiente para a realização de atividades de educação em saúde, com abordagens específicas para os grupos ocupacionais, atuando nas vulnerabilidades, com ações e monitoramento, visando desenvolvimento e qualidade ${ }^{42}$. Implantar ações de prevenção e controle da HA, atuando em seus fatores de risco, para reduzi-los, prevenir novos casos e controlar a doença nos já hipertensos valorizaria mais a saúde e o bem estar docente ${ }^{30}$. Assim, ações de prevenção e apoio direcionadas aos docentes, envolvendo também a gestão escolar, as famílias e a comunidade como um todo são recomendadas quando se almeja a maior valorização e melhor atuação dessa categoria profissional.

\section{Conclusão}

Este estudo verificou prevalência de 24,9\% de HA entre os docentes da educação básica, rede pública de um município brasileiro. Houve maior chance de HA entre os mais velhos, sem pós-graduação, atuantes também na rede privada, com maior consumo de sal, com sobrepeso/obesidade, com a RCQ elevada e com hipercolesterolemia/ hipertrigliceridemia e menor chance entre os do sexo feminino, que exerciam outra atividade de trabalho além da docência e insatisfeitos com o trabalho. Foi constatado que o perfil ocupacional docente exerce influência sobre a HA, mesmo após ajuste por variáveis reconhecidamente associadas à HA. O modelo teórico proposto mostrou-se pertinente e explicou 33,2\% da variabilidade do desfecho. Nesse contexto, ressaltase que a relação trabalho e saúde docente carece de atenção e cuidado, com a adoção de medidas preventivas, que objetivem sempre maior saúde e bem estar docente.

\section{Colaboradores}

MRM Vieira e DS Haikal trabalharam na concepção, coleta, análise e interpretação dos dados, redação do artigo. TA Magalhães, MM Vieira, VB Araújo, RRV Silva trabalharam na coleta de dados, análises e revisão. AMB de Paula e EF Ferreira atuaram na concepção e revisão crítica do artigo. 


\section{Agradecimentos}

Agradecimentos ao apóio logístico da Universidade Estadual de Montes Claros, à colaboração da Secretaria Estadual de Educação, às pessoas que aceitaram participar da pesquisa, às pessoas que contribuíram para a realização da pesquisa e às Agências de Fomento pela concessão de bolsas. MM Vieira é bolsita da Coordenação de Aperfeiçoamento de Pessoal de Nível Superior (CAPES), VB Araújo é Bolsista de IC da Fundação de Amparo à Pesquisa do Estado de Minas Gerais (FAPEMIG), AM Batista de Paula e EF Ferreira são Bolsistas de Produtividade em Pesquisa 2 pelo Conselho Nacional de Desenvolvimento Científico e Tecnológico (CNPq), DS Haikal é bolsista de produtividade da FAPEMIG.

\section{Referências}

1. Nóvoa A. Professores Imagens do futuro presente. Lisboa: Educa; 2009.

2. Jacomini MA, Penna MGO. Carreira docente e valorização do magistério: condições de trabalho e desenvolvimento profissional. Pro-Posições 2016; 27(2):177202.

3. Assunção AA. Educatel Brasil 2015/16: estimativas da frequência e distribuição dos principais condicionantes de saúde e de faltas ao trabalho na população de professores da educação básica no Brasil. Belo Horizonte: Ed do autor; 2016.

4. Felden Pereira É, Stefani Teixeira C, Pelegrini A, Meyer C, Andrade RD, Lopes AS. Estresse Relacionado ao Trabalho em Professores de Educação Básica. Ciencia \& trabajo 2014; 16(51):206-210.

5. Crescêncio D. Como vai a saúde dos nossos professores? Más condições no ambiente escolar impactam o desempenho profissional dos docentes e, consequentemente, o aprendizado alunos. In: Estadão - Blogs De olho na educação - Acompanhe de perto a educação no Brasil Todos pela educação. São Paulo: Estado de São Paulo; 2017.

6. Gasparini SM, Barreto SM, Assunção AA. O professor, as condições de trabalho e os efeitos sobre sua saúde. Educação e Pesquisa 2005; 31(2):189-199.

7. Batista JBV, Carlotto MS, Coutinho AS, Augusto LGS. Prevalência da Síndrome de Burnout e fatores sociodemográficos e laborais em professores de escolas municipais da cidade de João Pessoa, PB. Rev Bras Epidemiol 2010; 13(3):502-512.

8. Batista JBV, Carlotto MS, Moreira AM. Depressão como Causa de Afastamento do Trabalho: Um Estudo com Professores do Ensino Fundamental. Psico 2013; 44(2):257-262.

9. Brandão AA. Hipertensão Arterial. In: Rocha RM, Martins WA, organizadores. Manual de prevenção cardiovascular. São Paulo, Rio de Janeiro: Planmark, SOCERJ; 2017. p. 16-29.

10. VI Diretrizes Brasileiras de Hipertensão. Arquivos Brasileiros de Cardiologia 2010; 95:I-III.

11. Instituto Brasileiro de Geografia e Estatística (IBGE). Estimativas de população. Rio de Janeiro: IBGE; 2017.

12. Physical status: the use and interpretation of anthropometry. Report of a WHO Expert Committee. World Health Organ Tech Rep Ser 1995; 854:1-452.

13. Sturmer G, Dias-da-Costa JS, Olinto MTA, Menezes AMB, Gigante DP, Macedo S. O manejo não medicamentoso da hipertensão arterial sistêmica no Sul do Brasil. Cad Saude Publica 2006; 22(8):1727-1737.

14. Rosário TM, Scala LCN, França GVA, Pereira MR, Jardim PCBV. Fatores associados à hipertensão arterial sistêmica em Nobres-MT. Rev Bras Epidemiol 2009; 12(2):248-257.

15. Associação Brasileira de Empresas de Pesquisa (ABEP). Critério de Classificação Econômica Brasil. São Paulo: ABEP; 2014.

16. Brasil. Ministério da Saúde (MS). Brasil 2013: vigilância de fatores de risco e proteção para doenças crônicas por inquérito telefônico. Brasília: MS; 2014. 
17. Matsudo S, Araújo T, Matsudo V, Andrade D, Andrade E, Oliveira LC, Braggion G. Questionário internacional de atividade física (IPAQ): estudo de validade e reprodutibilidade no Brasil. Atividade Física \& Saúde 2001; 6(2):5-18.

18. Conti MA, Jardim AP, Hearst N, Cordás TA, Tavares $\mathrm{H}$, Abreu CN. Avaliação da equivalência semântica e consistência interna de uma versão em português do Internet Addiction Test (IAT). Rev. Psiquiatr. Clín. 2012; 39(3):106-110.

19. Fleck MP, Louzada S, Xavier M, Chachamovich E, Vieira G, Santos L, Pinzon V. Aplicação da versão em português do instrumento abreviado de avaliação da qualidade de vida "WHOQOL-bref”. Rev Saude Publica 2000; 34(2):178-183.

20. Paiva CRE, Gaya ACA, Bottaro M, Bezerra RFA. Assessment of the body composition of brazilian boys: the bioimpedence method. Rev. Bras. Cineantropom. Desempenho Hum 2002; 4(1):9.

21. Brasil. Ministério da Saúde (MS). Brasil 2012: vigilância de fatores de risco e proteção para doenças crônicas por inquérito telefônico. Brasília: MS; 2013.

22. Gorestein C, Andrade LHSG. Inventário de depressão de Beck: propriedades psicométricas da versão em português. Rev Psiq Clin 1998; 25(5):245-250.

23. Cunha J. Manual em português das Escalas Beck. São Paulo: Casa do Psicólogo; 2001.

24. Lipp MEN. Manual do inventário de stress para adultos de Lipp. São Paulo: Casa do Psicólogo; 2005.

25. Gil-Monte PR, Moreno-Jiménez B. El síndrome de quemarse por eltrabajo ("Burnout"): Una enfermedad laboral enla sociedade delbienestar. Madrid: Pirámide; 2005.

26. Silva NN. Amostragem Probabilística: Um Curso Introdutório. Sao Paulo: Editora da Universidade de São Paulo; 2004.

27. Brasil. Ministério da Saúde (MS). Brasil 2016: vigilância de fatores de risco e proteção para doenças crônicas por inquérito telefônico: estimativas sobre frequência e distribuição sociodemográfica de fatores de risco e proteção para doenças crônicas nas capitais dos 26 estados brasileiros e no Distrito Federal em 2016. Brasília: MS; 2017.

28. Moreira OC, Oliveira RAR, Andrade Neto F, Amorim W, Oliveira CEP, Doimo LA, Amorim PRS, Laterza MC, Monteiro WD, Marins JCB. Associação entre risco cardiovascular e hipertensão arterial em professores universitários. Revista Brasileira de Educação Física e Esporte 2011; 25(3):397-406.

29. Santos MN, Marques AC. Condições de saúde, estilo de vida e características de trabalho de professores de uma cidade do sul do Brasil. Cien Saude Colet 2013; 18(3):837-846.

30. Oliveira RAR, Mota Júnior RJ, Tavares DDF, Moreira $\mathrm{OC}$, Marins JCB. Fatores associados à pressão arterial elevada em professores da educação básica. Revista da Educação Física / UEM 2015; 26(1):119-129.

31. Rodrigues ME, Doimo LA, Marins JCB. Perfil pressórico entre estudantes, professores e funcionários de uma universidade pública. J Health Sci Inst 2011; 29(1):56-61.
32. Silva CS, Paes NA, Figueiredo TMRM, Cardoso MAA, Silva ATMC, Araújo JSS. Controle pressórico e adesão/vínculo em hipertensos usuários da Atenção Primária à Saúde. Revista da Escola de Enfermagem da USP 2013; 47(3):584-590.

33. Zangirolani LTO, Assumpção D, Medeiros MAT, Barros MBA. Hipertensão arterial autorreferida em adultos residentes em Campinas, São Paulo, Brasil: prevalência, fatores associados e práticas de controle em estudo de base populacional. Cien Saude Colet 2018; 23(4):1221-1232.

34. Malta DC, Bernal RTI, Andrade SSCA, Silva MMA, Velasquez-Melendez G. Prevalence of and factors associated with self-reported high blood pressure in Brazilian adults. Rev Saude Publica 2017; 51(Supl. 1):11S

35. Guedes AMA, Lima DF, Abreu EF, Sousa GMC. Mal -estar docente: Quando a prática compromete a saude do professor. REVASF 2013; 2(2):44-54.

36. Marqueze EC, Moreno CRC. Satisfação no trabalho e capacidade para o trabalho entre docentes universitários. Psicologia em Estudo 2009; 14(1):75-82.

37. Zille LP, Cremonezi AM. Estresse no trabalho: Estudo com professores da rede pública estadual de Minas Gerais. REUNA 2013; 18(4):111-128.

38. Cassettari N, Scaldelai VDF, Frutuoso PC. Exoneração a Pedido de Professores: estudo em duas redes municipais paulistas. Educação \& Sociedade 2014; 35(128):909-927.

39. Keller N, Krummel T, Hannedouche T. Sodium, hypertension, maladies rénales et santé publique. Néphrologie \& Thérapeutique 2018; 14(Supl.):S93-S98

40. Sarno F, Claro RM, Levy RB, Bandoni DH, Monteiro CA. Estimativa de consumo de sódio pela população brasileira, 2008-2009. Rev Saude Publica 2013; 47(3):571-578.

41. Peixoto MDR, Morais Cordeiro M, Roriz Ferreira V, Cardoso CKS, Crispim P. Ganho de peso na vida adulta: preditor da hipertensão arterial? Cad. Saúde Colet 2017; 24(1):58-64.

42. Freitas PP, Assunção AA, Bassi IB, Lopes ACS. Excesso de peso e ambiente de trabalho no setor público municipal. Revista de Nutrição 2016; 29(4):519-527.

43. Guilbert JJ. The world health report 2002 - reducing risks, promoting healthy life. Educ Health 2003; 16(2):230.

44. Radovanovic CAT, Bevilaqua CA, Molena-Fernandes CA, Marcon SS. Intervenção multiprofissional em adultos com hipertensão arterial: ensaio clínico randomizado. Revista Brasileira de Enfermagem 2016; 69:1067-1073.

Artigo apresentado em 07/05/2018

Aprovado em 03/10/2018

Versão final apresentada em 05/10/2018 
